\title{
Tryptophan metabolism is differently regulated between large and small dogs
}

\author{
Jessica M. Hoffman (D) - J. Veronika Kiklevich • \\ Marika Austad • ViLinh Tran • Dean P. Jones • \\ Angela Royal • Carolyn Henry • Steven N. Austad
}

Received: 19 June 2019 / Accepted: 2 October 2019 / Published online: 29 November 2019

(C) The Author(s) 2019

\begin{abstract}
Companion dogs have recently been promoted as an animal model for the study of aging due to their similar disease profile to humans, the sophistication of health assessment and disease diagnosis, and the shared environments with their owners. In addition, dogs show an interesting life history trait pattern where smaller individuals are up to two-fold longer lived than their larger counterparts. While some of the mechanisms underlying this size and longevity trade-off are strongly suspected (i.e., growth hormone/IGF-I), there are likely a number of undiscovered mechanisms as well. Accordingly, we have completed a large-scale global metabolomic profiling of dogs encompassing a range of sizes and ages from three cities across the USA. We
\end{abstract}

Electronic supplementary material The online version of this article (https://doi.org/10.1007/s11357-019-00114-x) contains supplementary material, which is available to authorized users.

J. M. Hoffman $(\bowtie) \cdot J$. V. Kiklevich $\cdot$ M. Austad •

S. N. Austad

Department of Biology, University of Alabama at Birmingham, 1300 University Blvd. CH464, Birmingham, AL 35294, USA e-mail:.jmhoffm@uab.edu

V. Tran · D. P. Jones

Division of Pulmonary Allergy and Critical Care, Department of Medicine, Emory University, Atlanta, GA, USA

V. Tran · D. P. Jones

Clinical Biomarkers Laboratory, Department of Medicine, Emory University, Atlanta, GA, USA

A. Royal · C. Henry

Veterinary Medical Diagnostic Laboratory, University of Missouri, 900 E Campus Dr, Columbia, MO 65211, USA found a surprisingly strong location signal in the metabolome, stronger in fact than any signal related to age, breed, or sex. However, after controlling for the effects of location, tryptophan metabolism emerged as significantly associated with weight of the dogs, with small dogs having significantly higher levels of tryptophan pathway metabolites. Overall, our results point toward novel, testable hypotheses about the underlying physiological mechanisms that influence size and longevity in the companion dog and suggest that dogs may be useful in sorting out the complexities of the tryptophan metabolic network.

Keywords Dog $\cdot$ Metabolomics $\cdot$ Body size . Tryptophan metabolism

\section{Introduction}

Humans are living longer than ever before in the 300thousand-year history of our species. Consequently, we often spend our later years battling frailty, disability, and/or multiple life-threatening illnesses. To begin to understand the underlying aging processes that so often degrade the quality of later life, model laboratory organisms such as Caenorhabditis elegans, fruit flies, and mice are most commonly employed with an ultimate goal of discovering interventions that can be translated to improve and extend human health. However, translation of findings from these common laboratory species to effective interventions in humans has been uncertain (Cheon and Orsulic 2011; von Scheidt et al. 2017). 
Among the reasons for this limited therapeutic translatability are (1) overreliance on one or a few genetic backgrounds, (2) model species accumulate very different late-life pathologies compared with humans, and (3) they are typically maintained in highly controlled, unchanging, specific pathogen-free, benign environments that bear little resemblance to the range of environments that humans inhabit. To address these issues, the companion dog has recently been promoted as a more representative model of human health and longevity (e.g., Hoffman et al. 2018a; Kaeberlein et al. 2016). Compared with laboratory mice, for instance, companion dogs are genetically heterogeneous, experience an extraordinarily similar range of late-life pathologies as humans, and of course share the same environments as their owners. In addition, because of the sophistication of veterinary science, their health status can be exquisitely monitored and pathologies identified with precision possibly only second to that of humans (e.g., Jin et al. 2016). In this regard, dogs have been found to have age-related degenerative valve disease (Urfer et al. 2017a) as well as reduced oral health with aging (An et al. 2018), both similar to humans. In a similar vein, dogs have recently been proposed as a model to study lifespan extending interventions, and early work with rapamycin supplementation showed no negative health effects with a potential improvement in heart function in a small cohort of dogs (Urfer et al. 2017b). While these studies all suggest the power of the dog as a model of translational human aging, little is known about the molecular changes that occur with, and contribute to, aging in the dog.

In addition to their strengths as a model of human aging and longevity, dogs show a potentially highly informative pattern relating body size and longevity. Across mammalian species, larger species tend to be longer lived (Healy et al. 2014); however, within a species, like the companion dog, smaller individuals are longer lived. This pattern is seen in other mammalian species that show a significant size variation including mice and rats (Miller et al. 2002; Rollo 2002), horses (Tapprest et al. 2017), and possibly humans (He et al. 2014; Ma et al. 2017), although data conflict on this last point with some studies observing that taller individuals live longer (e.g., Brandts and van den Brandt 2019). However, no other species is known to show the body size-longevity relationship to the extent of the companion dog, in which a 50 -fold variation in body mass is negatively associated with a 2 -fold range in lifespan
(Fleming et al. 2011; O'Neill et al. 2013). Thus, weight can be used an approximation of longevity, as smaller dogs are expected to live longer than larger ones.

The physiological parameters that contribute to the size-longevity trade-off seen in mammals is multi-faceted. Growth hormone $(\mathrm{GH})$ and IGF-I signaling are almost certainly involved. In mice, it is well established that reducing GH signaling, either by lowering hormone availability or by disrupting its receptor, increases longevity and preserves multiple dimensions of health (Bartke 2005). Consistent with these findings, in dogs, GH and circulating IGF-1 levels are higher in large compared with medium-sized dog breeds (Favier et al. 2001; Greer et al. 2011). In addition to the GH/IGF axis, recent research using cultured fibroblasts from small and large dogs suggests that differences in mitochondrial metabolism and oxidative stress might contribute to the longevity differences seen across different-sized dogs. Cells from large dogs have significantly higher rates of glycolysis and DNA damage (Jimenez et al. 2018), as well as higher mitochondrial respiration rates (Nicholatos et al. 2019). However, many of the underlying mechanisms that contribute to longevity in the dog are still unknown. By investigating size-longevity differences, we can begin to develop novel hypotheses about healthy aging that potentially can be translated to human (and dog) life-extending interventions.

One method to identify molecular networks underlying complex physiological processes is metabolomics, the analysis of thousands of individual metabolites in an organism to understand how changes in metabolism are associated with specific phenotypes of interest. Metabolomics has been used extensively in model organisms to understand aging and longevity (e.g., Fuchs et al. 2010; Hoffman et al. 2014; Houtkooper et al. 2011), as well as non-human primates (Hoffman et al. 2018b; Hoffman et al. 2016) and humans (Darst et al. 2019; Menni et al. 2013). Previous research has suggested that individual dog breeds show different metabolomic profiles (Lloyd et al. 2016; Nicholatos et al. 2019; Viant et al. 2007) and that metabolomic profiles change in response to diet and obesity (Forster et al. 2018; Soder et al. 2017), as well as with specific diseases (e.g., Gookin et al. 2018; Hasegawa et al. 2014; Minamoto et al. 2015). Dogs of varying sizes have been shown to have different levels of circulating amino acids (Middleton et al. 2017), as well as different metabolomic profiles (Nicholatos et al. 2019). 
Here, we present the largest metabolomics study to date in the companion dog with the goal of developing novel hypotheses about mechanisms of canine aging and longevity. We specifically look at the dog metabolome sampled at three different locations across the USA to help assess the impact of environmental heterogeneity.

\section{Methods}

Sample collection

Whole blood from animals undergoing unrelated procedures was collected in EDTA tubes from companion dogs in three locations between 2016 and 2018: (1) Birmingham, AL; (2) San Antonio, TX; and (3) Columbia, MO. Birmingham samples were collected from stray animals brought into the Jefferson County animal control facility and generally healthy animals seen at local veterinary clinics under the direction of JVK. San Antonio samples were collected by JVK and MA from generally healthy animals belonging to private owners brought to a local spay/neuter clinic. Columbia samples were collected as part of diagnostic workups for Veterinary Health Center patients, with sample processing coordinated by $\mathrm{AR}$ and $\mathrm{CH}$. Individuals from this location included acute and chronically ill animals in addition to apparently healthy animals. A majority of samples from Birmingham and Columbia were from dogs fasted at least 4-6 h before blood collection. The San Antonio samples were from animals fasted overnight before the day of collection. During blood draw, demographic information for each dog were recorded: age, sex, sterilization status, body weight, and body condition score (BCS), a measure of obesity in the dog. Breed of dog was assigned either by the tending veterinarian or by the owner of the dog (Table S1). Age of stray animals from the Jefferson County animal control facility was estimated by JVK from a combination of bone development, and dental and ocular characteristics. Collection of blood samples from person-owned dogs was approved under UAB IACUC 21121 and MU ACUC 8240.

After sample collection, tubes were stored at $4{ }^{\circ} \mathrm{C}$. Plasma was extracted by centrifugation and then frozen at $-80{ }^{\circ} \mathrm{C}$ until plasma metabolite extraction. Samples from the three collection sites were randomized to minimize batch effects during metabolomics analyses and shipped on dry ice to the Clinical Biomarkers Laboratory, Emory University, for analysis.

Metabolomics

High-resolution metabolomics (HRM) profiling was completed using standardized methods (Liu et al. 2016; Soltow et al. 2013) as follows. The 133 samples were analyzed in three batches consisting of 44,44 , and 45 samples, each prepared daily along with pooled human plasma (Qstd3) for quality control. For analysis prior to the first and after the last batch, an additional aliquot of National Institute of Standards and Technology Standard Reference Material 1950 (NIST SRM1950) was processed and analyzed identically to the samples. Aliquots were removed from storage at $80^{\circ} \mathrm{C}$, thawed on ice, and $50 \mu \mathrm{L}$ was treated with 100 $\mu \mathrm{L}$ of ice-cold LC-MS-grade acetonitrile. Extracts were then equilibrated for $30 \mathrm{~min}$ on ice, centrifuged $\left(16,100 \times g\right.$ at $\left.4{ }^{\circ} \mathrm{C}\right)$ for $10 \mathrm{~min}$ to remove precipitated proteins, and clear supernatant was transferred to $250-\mu \mathrm{L}$ autosampler vials and maintained at $4{ }^{\circ} \mathrm{C}$ until analysis $(<22 \mathrm{~h})$.

Sample extracts were analyzed using liquid chromatography and Fourier transform high-resolution mass spectrometry on a Dionex Ultimate 3000, Orbitrap Fusion $^{\mathrm{TM}}$ Tribrid $^{\mathrm{TM}}$ Mass Spectrometer system (Thermo Scientific) operated at 120,000 resolution. The chromatography system was operated in a dualpump configuration that enabled parallel analyte separation and column flushing. For each sample, $10 \mu \mathrm{L}$ aliquots were analyzed in triplicate using hydrophilic interaction liquid chromatography (HILIC) with electrospray ionization (ESI) source operated in positive mode and reverse-phase chromatography (RPC) with ESI operated in negative mode. Analyte separation for HILIC was accomplished by a $2.1 \mathrm{~mm} \times 50 \mathrm{~mm} \times$ $2.5 \mu \mathrm{m}$ Waters XBridge BEH Amide XP HILIC and an eluent gradient $(\mathrm{A}=$ water, $\mathrm{B}=$ acetonitrile, $\mathrm{C}=2 \%$ formic acid) consisting of an initial 1.5-min period of $22.5 \% \mathrm{~A}, 75 \% \mathrm{~B}$, and $2.5 \% \mathrm{C}$ followed by a linear increase to $77.5 \% \mathrm{~A}, 20 \% \mathrm{~B}$, and $2.5 \% \mathrm{C}$ at $4 \mathrm{~min}$ and a final hold of $1 \mathrm{~min}$. RPC separation was by $2.1 \mathrm{~mm} \times$ $50 \mathrm{~mm} \times 3 \mu \mathrm{m}$ end-capped $\mathrm{C}_{18}$ column (Higgins) using an eluent gradient $(\mathrm{A}=$ water, $\mathrm{B}=$ acetonitrile, $\mathrm{C}=$ $10 \mathrm{mM}$ ammonium acetate) consisting of an initial 1min period of $60 \% \mathrm{~A}, 35 \% \mathrm{~B}$, and $5 \% \mathrm{C}$, followed by a linear increase to $0 \% \mathrm{~A}, 95 \% \mathrm{~B}$, and $5 \% \mathrm{C}$ at $1.5 \mathrm{~min}$ and held for the remaining $3.5 \mathrm{~min}$. The mobile phase 
flow rate for HILICpos was held at $0.350 \mathrm{~mL} / \mathrm{min}$ for the first $1.5 \mathrm{~min}$, and increased to $0.400 \mathrm{~mL} / \mathrm{min}$ for the remaining of the run. $\mathrm{C} 18$ neg mobile phase flow rate was held at $0.400 \mathrm{ml} / \mathrm{min}$ for the first $2 \mathrm{~min}$ and then increased to $0.500 \mathrm{ml} / \mathrm{min}$ for the remaining $3.0 \mathrm{~min}$. Data were collected for a mass-to-charge ratio $(\mathrm{m} / \mathrm{z})$ range $85-1275$. Probe temperature, capillary temperature, sweep gas, and S-Lens RF levels were maintained at $200{ }^{\circ} \mathrm{C}, 300{ }^{\circ} \mathrm{C}, 1$ arbitrary units (AU), and 45 , respectively, for both ESI polarities. Additional source tune settings were optimized for sensitivity using a standard mixture; positive tune settings for sheath gas, auxiliary gas, sweep gas, and spray voltage setting were $45 \mathrm{AU}, 25 \mathrm{AU}, 1 \mathrm{AU}$, and $3.5 \mathrm{kV}$, respectively; negative settings were $45 \mathrm{AU}, 5 \mathrm{AU}, 1 \mathrm{AU}$, and $-3.0 \mathrm{kV}$. Maximum C-trap injection times of $100 \mathrm{~ms}$ and automatic gain control target of $1 \times 10^{6}$ for both polarities. During untargeted data acquisition, no exclusion or inclusion masses were selected, and data were acquired in $\mathrm{MS}^{1}$ mode only. Data were stored as .raw files and converted to CDF format using Xcalibur file converter software (Thermo Fisher, San Diego, CA) for further data processing. Peak detection, noise filtering, $\mathrm{m} / \mathrm{z}$ and retention time alignment, feature quantification, and data quality filtering were performed using apLCMS (Yu et al. 2009) with xMSanalyzer (Uppal et al. 2013). Data were extracted as $\mathrm{m} / \mathrm{z}$ features where a feature is defined by $\mathrm{m} / \mathrm{z}$, retention time, and integrated ion intensity.

As a confirmation of metabolite values in our dataset, we compared individual metabolite values with known standards (Qstd3 as described above). This was done by taking the known Qstd3 value and multiplying it by metabolite intensity of each canine sample divided by the mean Qstd3 value for that metabolite. This gave us a "true" concentration of the metabolite in the canine samples compared with a human reference.

\section{Data analysis}

Metabolomics data analysis was completed in the statistical language $\mathrm{R}$ unless otherwise stated ( $\mathrm{R}$ Core Team 2018). Positive and negative ion mode data were analyzed separately. All data were first log-transformed and centered and scaled to using the "scale" function in R. Metabolites that were missing from more than $15 \%$ of all samples were removed from the analysis. Dogs who were recorded as being under 1 year of age were removed from the analysis as final body size had not yet been attained.

Our initial interest was in determining the association between individual metabolites, weight, and age, controlling for the effects of sex and location. We also investigated the effect of sterilization status on the metabolome and found little effect. Thus, sterilization status was dropped from our final model. Significance was set with a false discovery rate (FDR) of $\alpha<0.05$ (Benjamini and Hochberg 1995). Metabolites that were found to be associated with weight or age were run through the program mummichog to determine metabolic pathways that were significantly different for each factor individually (Li et al. 2013). We did not control or look at the impact of breed in our model as there were over 20 breeds represented in our final dataset. Therefore, we did not have the power to assess breedspecific differences.

In addition to our individual metabolite analyses, we examined the associations of the entire metabolome with sex, age, and body weight. Principal components analysis (PCA) was performed using the ade4 package in R (Dray and Dufour 2007), for only those metabolites that were present in all samples.

\section{Results}

Our final dataset consisted of plasma samples from 112 dogs across the three locations (44-Birmingham, 38Columbia, 30-San Antonio). The mean age of all animals from all sites was 5.5 years (1-17 years) with an average weight of $19.7 \mathrm{~kg}(2.1-76.4 \mathrm{~kg})$. Males slightly outnumbered our female samples (62 males, 50 females); $49 \%$ of females and $55 \%$ of males were sterilized at the time of sample collection. Clear differences were noted in the characteristics of the dogs among the three collection sites (Fig. 1). Dogs from Columbia were overall older and largely sterilized compared with younger, smaller 100\% intact dogs from San Antonio. In fact, dogs from San Antonio were smaller than those from either of the other two locations. In addition, $0 \%, 18.2 \%$, and $71 \%$ of dogs at San Antonio, Birmingham, and Columbia had a serious health diagnosis at the time of sample draw (Table S1). Thus, the dogs sampled in Columbia were on average sicker than the other two populations. Therefore, all three locations represent very different canine populations. 

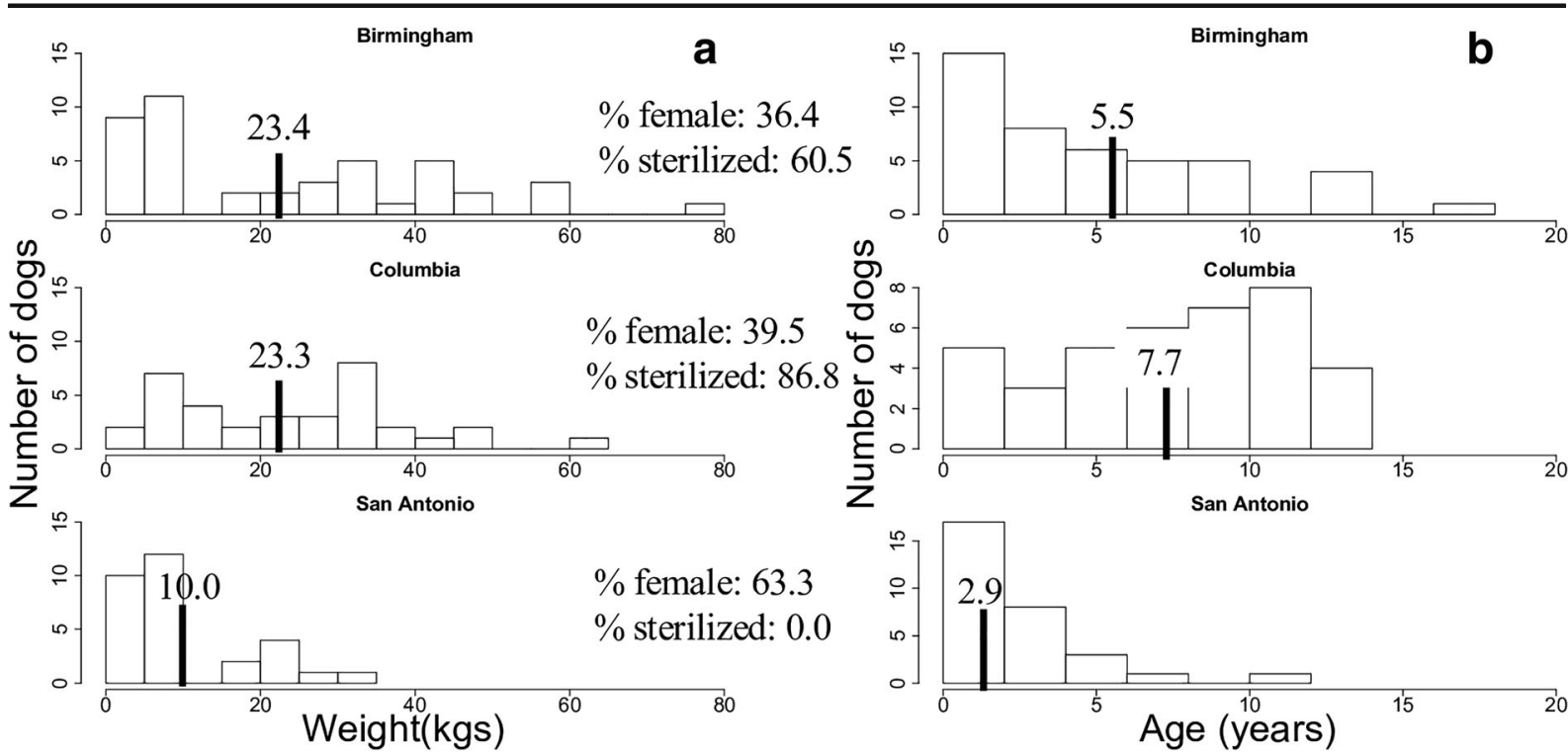

Fig. 1 Profile of dogs sampled from the three locations. (a) Weight. (b) Age. Note that vertical lines indicate population mean.

Our final metabolomics dataset consisted of 6789 metabolites in the positive ion mode and 6614 metabolites in the negative ion mode. Our residual linear regression model and PCA comprised 3473 and 3442 metabolites in the positive and negative ion modes, respectively; this difference in metabolite number is due to including only those metabolites that were found to have no missing data across all samples in the analyses.

We found that, perhaps not surprisingly, location had the biggest association with the metabolome. The metabolomes of San Antonio dogs were most different from Birmingham and Columbia (Figure 2). This location effect was so strong that it overwhelmed associations of individual metabolites with our factors of interest as little variation was left in the dataset (Table 1). To control this location effect, we utilized two different approaches. In the first, we took the residuals of each metabolite by location, and then used the residual values in a linear model. Secondly, we analyzed each location individually, comparing significant metabolites across locations.

Using the residuals of location, we were able to discover 161 and 293 metabolites associated with age and 59 and 32 metabolites associated with weight in the positive and negative ion modes, respectively (Table 2). We found very few metabolites associated with sex, 1 and 11 in the positive and negative modes, respectively.
Percent of the sample that is female and that comes from sterilized dogs shown for each location

However, our PCA failed to separate the metabolome based on either age or weight (Figure S1). Metabolite enrichment analyses discovered 9 and 5 pathways associated with weight, and 8 and 31 pathways associated with age in the positive and negative ion modes, respectively (Table 3). We found a strong signal for differences in tryptophan metabolism between large and small dogs, with higher values of tryptophan metabolites always seen in the small dogs (Fig. 3a-f). However, tryptophan itself was not higher in small dogs (Fig. 3g), nor was kynurenine, its immediate metabolized breakdown product (Fig. 3h). We then quantified tryptophan using the raw intensity values and found that levels of tryptophan varied from 6.55-132.12 and showed a similar pattern with body weight and the normalized residual values in the model (Figure S2).

When we looked within individual locations, we were actually able to find more metabolites associated with age and weight, even though our power was significantly decreased with the reduction in sample size in a location-specific analysis. Striking differences were seen in the numbers of metabolites associated with each factor of interest. Specifically, over $13 \%$ of the metabolome was associated with age in our Birmingham samples, but less than $1 \%$ were associated with age in San Antonio. This is not surprising as San Antonio had the smallest age variation (Fig. 1). No metabolites passed our FDR cutoff in our Columbia samples. Weight was 
a

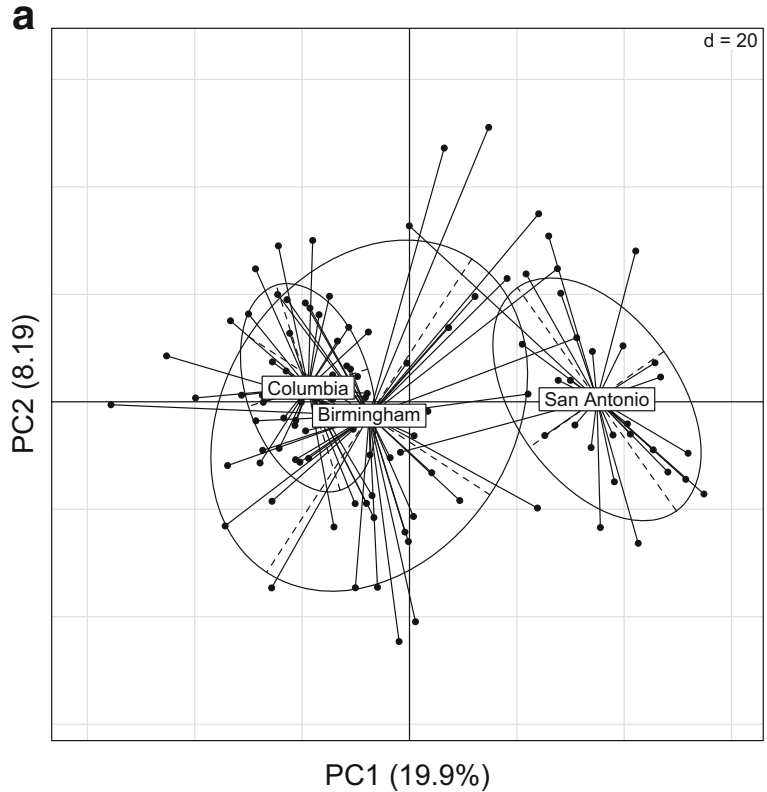

b

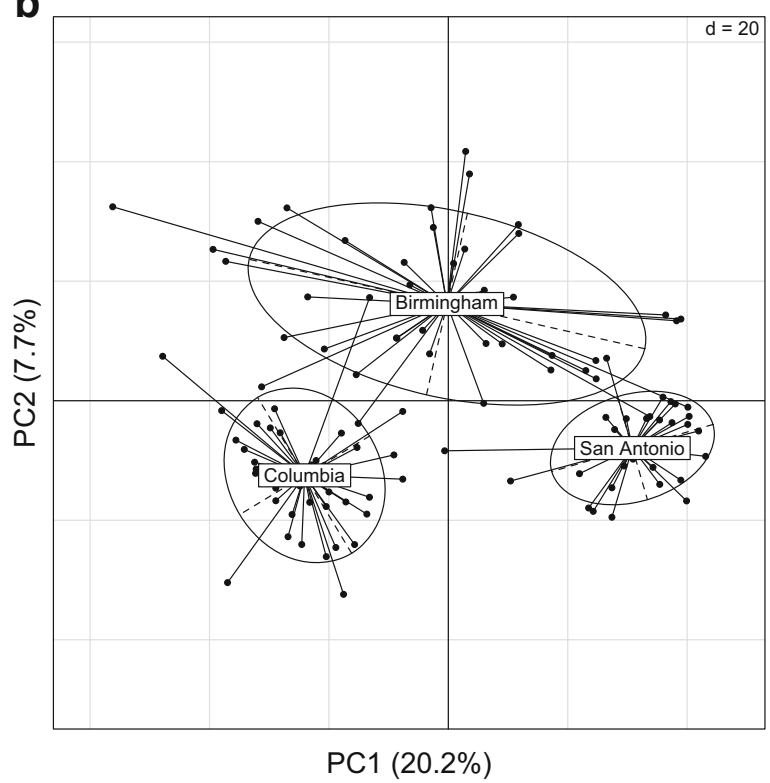

Fig. 2 PCA effects of location for the a positive and $\mathbf{b}$ negative ion modes

associated with just over $1 \%$ of metabolites in both Birmingham and San Antonio, and again, no metabolites passed our FDR cutoff in Columbia. Sex was significantly associated with metabolite concentration only in the San Antonio location and only in the negative ion mode. The metabolites associated with age and weight in Birmingham and San Antonio rarely overlapped.

For those metabolites that were associated with either weight or age in Birmingham, we ran metabolite enrichment analysis to determine which metabolic pathways were differentially associated with which variable. Similar to the entire combined datasets, the strongest differences between large and small dogs were related to tryptophan metabolism. Specifically, in the positive ion mode, 6 different metabolites were negatively associated with body size (Fig. 4). These findings replicate those seen in the location residual performed previously,

Table 1 Number of metabolites associated with age, sex, and weight across all locations. Linear model has controlled for the effects of location. Models were run with 3473 and 3442 metabolites in the positive and negative ion modes, respectively (all metabolites with no missing data)

\begin{tabular}{lccc}
\hline Column & Age & Sex & Weight \\
\hline Hilic positive & 161 & 1 & 59 \\
C18 negative & 293 & 11 & 32 \\
\hline
\end{tabular}

suggesting many of the effects seen in the entire dataset are driven partially at least by the differences in the Birmingham location.

In addition to the differences in tryptophan metabolism, urea cycle metabolism, and metabolism of fatty acids, linoleate metabolism, de novo fatty acid synthesis, fatty acid activation, and vitamin A metabolism were associated with weight, while fatty acid metabolism and arginine, proline, and alanine metabolism were associated with age (Table 2). Many of the metabolic pathways associated with age and weight in the Birmingham sample were also significantly enriched in the entire dataset when using residuals of location as described above (Table 2).

Table 2 Number of metabolites associated with age, sex, and weight for each location individually. Models were run with 6789 and 6614 metabolites in the positive and negative ion modes, respectively

\begin{tabular}{lllll}
\hline Column & Location & Age & Sex & Weight \\
\hline Hilic positive & Birmingham & 930 & 0 & 106 \\
& Columbia & 0 & 0 & 0 \\
& San Antonio & 26 & 4 & 101 \\
C18 negative & Birmingham & 1474 & 0 & 90 \\
& Columbia & 0 & 0 & 0 \\
& San Antonio & 13 & 45 & 21 \\
\hline
\end{tabular}




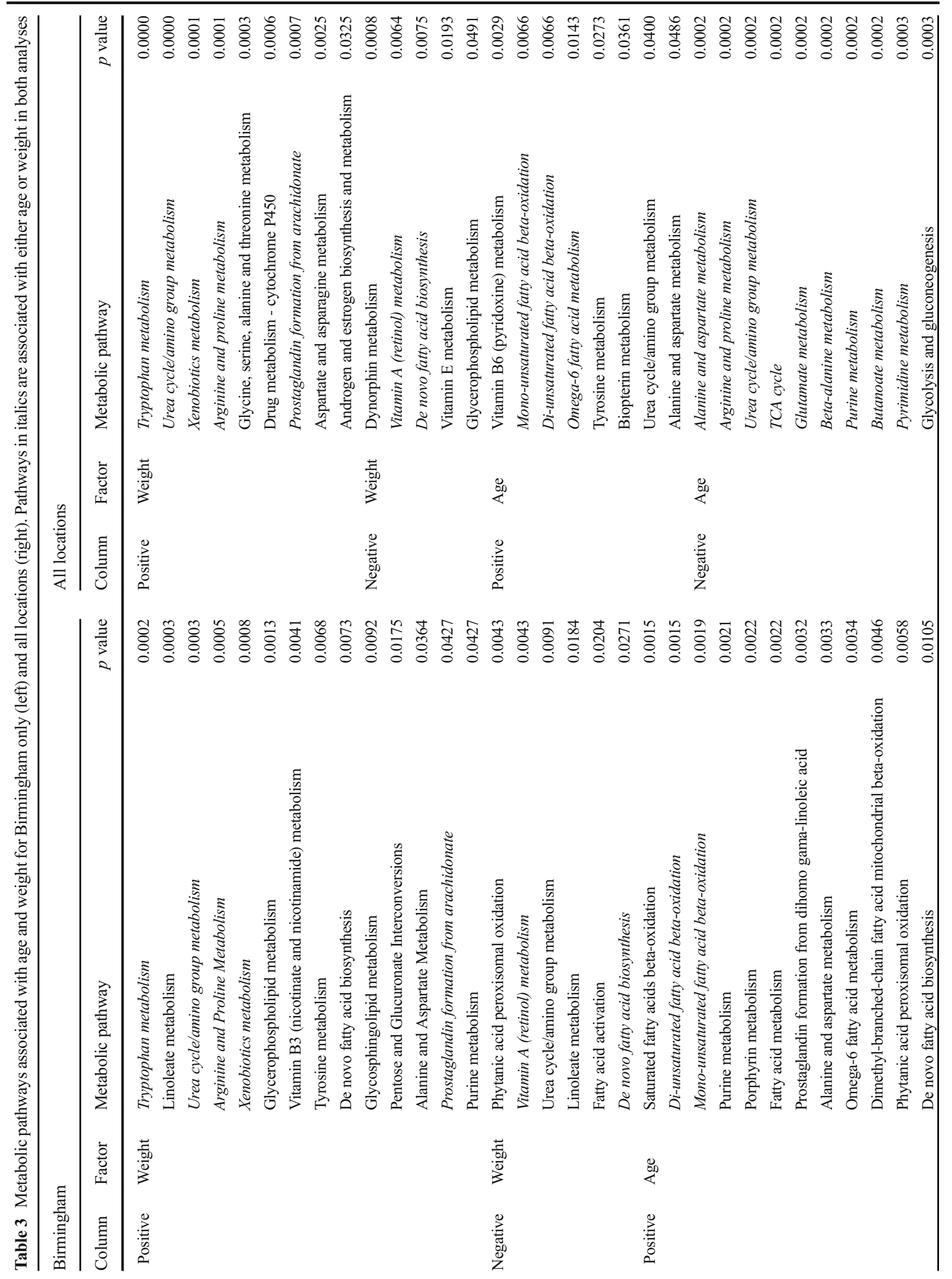




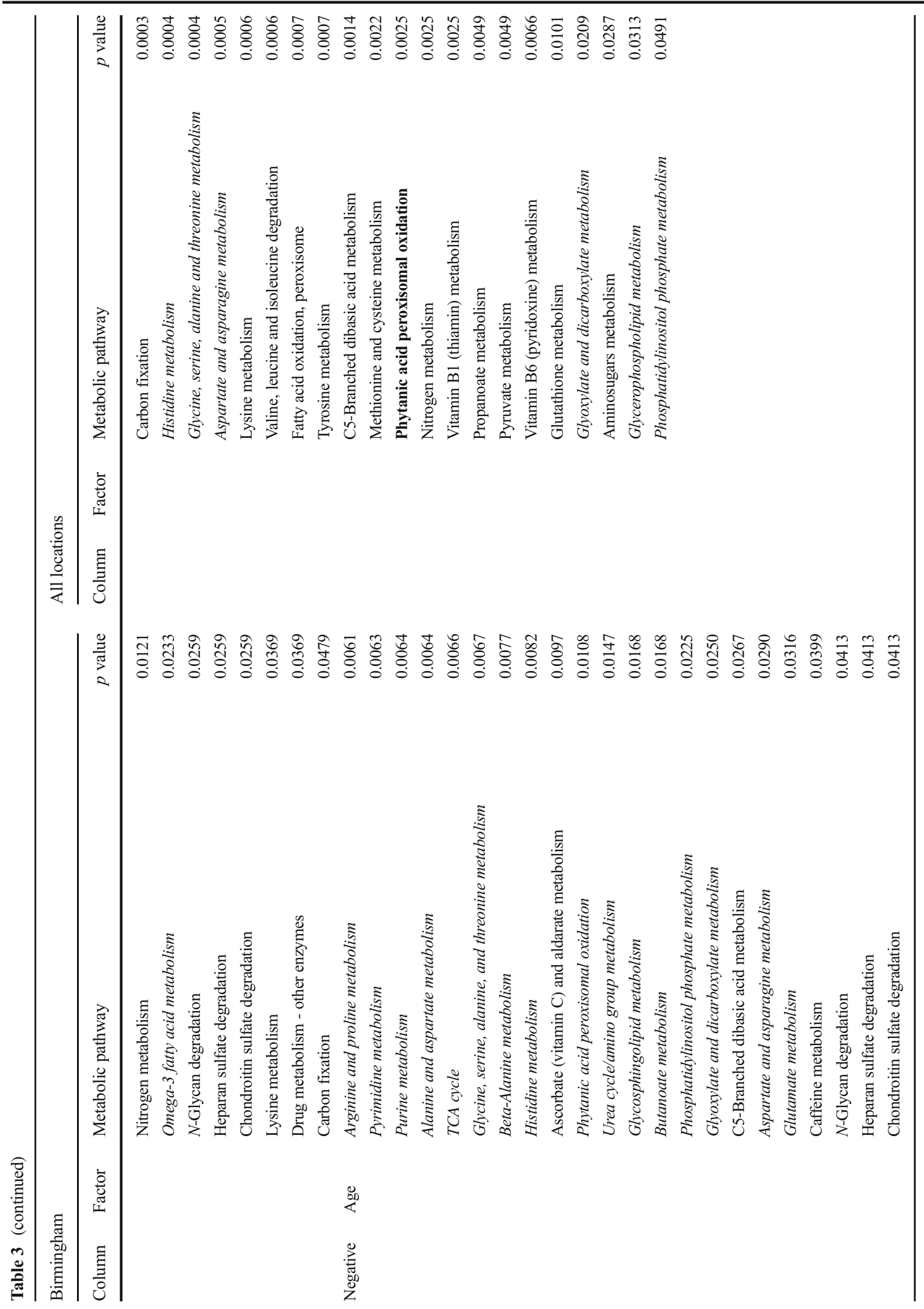



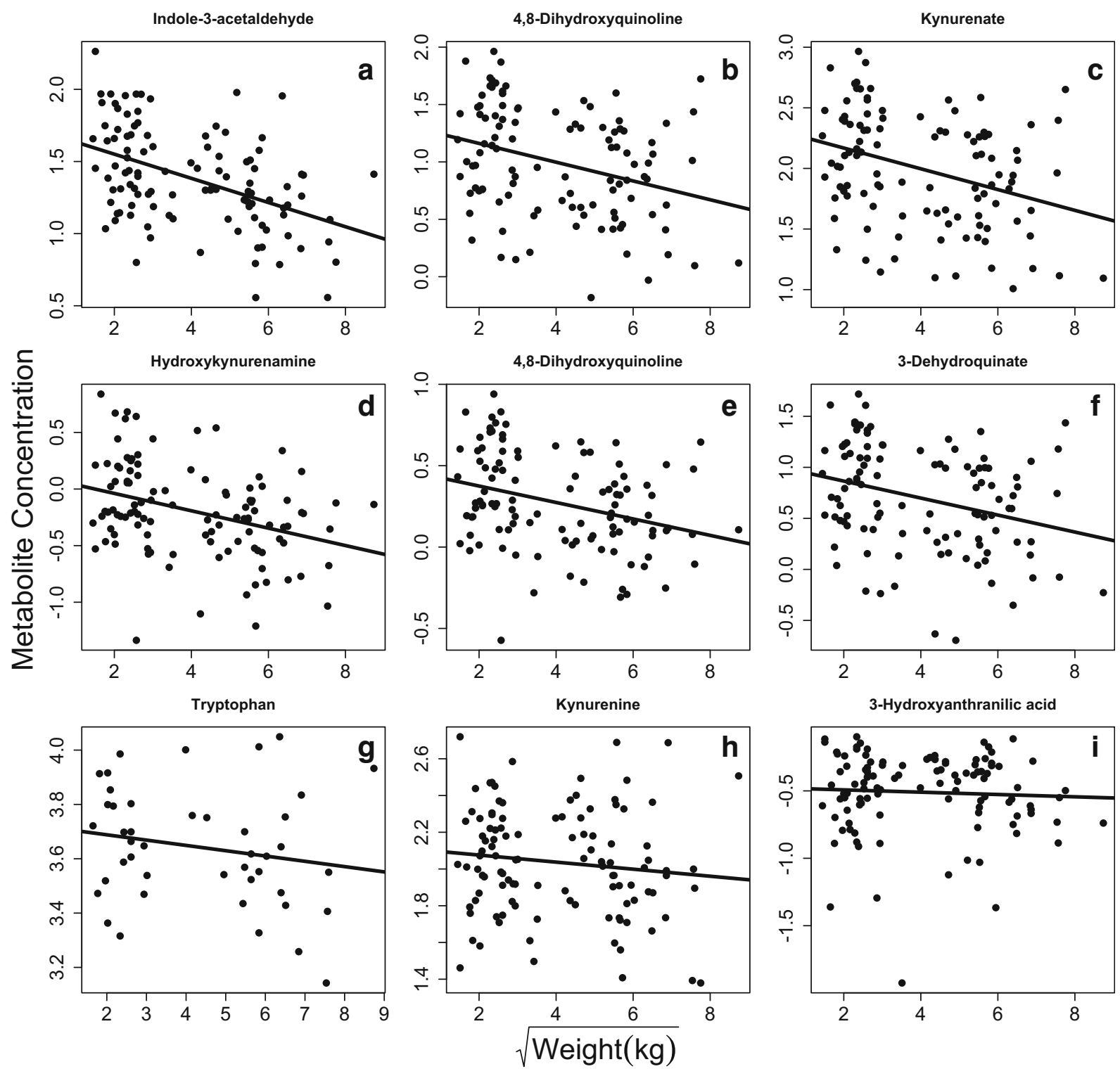

Fig. 3 Tryptophan metabolites associated with weight across all location. a-f All were significantly associated with weight at FDR $<0.05$. g-i Other annotated metabolites in the pathway that did not pass our FDR threshold. All significant metabolites are lower in larger dogs, controlling for the effects of age and sex. Note that weight has been square-root transformed to make visualization easier

the two group in a PCA analysis (Fig. 5). However, running a linear model with cancer status failed to find any metabolites that passed our FDR cutoff.

\section{Discussion}

Here, we have completed the largest metabolomic profiling study to date in the companion dog, as well as the ion mode, cancer status was able to somewhat separate 

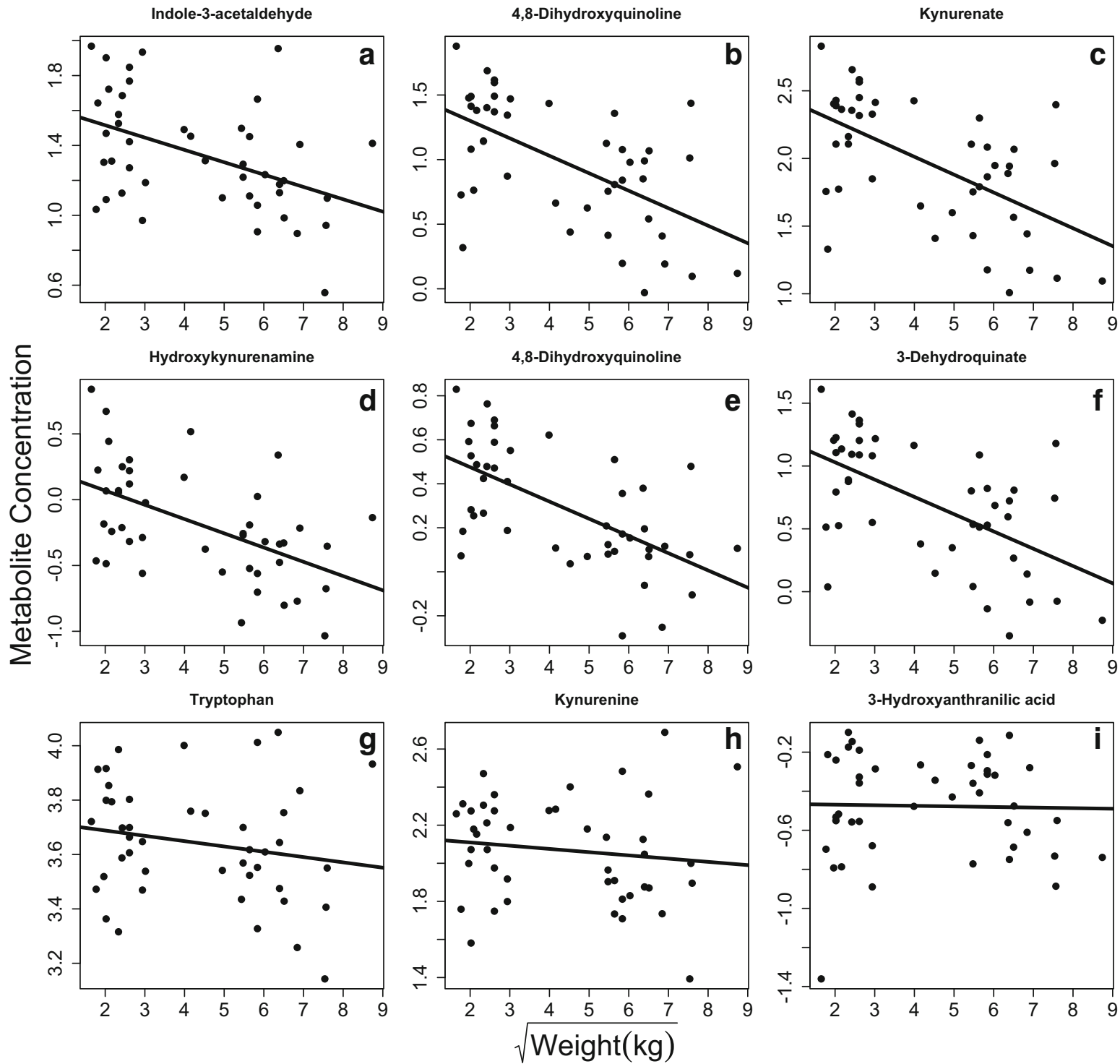

Fig. 4 Tryptophan metabolites associated with weight in Birmingham only. a-f All were significantly associated with weight at FDR $<0.05$. g-i Other annotated metabolites in the pathway that did not pass our FDR threshold. All significant metabolites are

first to examine how the metabolome changes with age and weight. Interestingly, the largest effect we found was with regard to location of sampling. This large effect was quite unexpected, especially considering two of the locations (Birmingham and San Antonio) had samples drawn by the same veterinarian into EDTA tubes from the same shipment. However, the age, size, and reproductive status distributions of these two populations differed dramatically, so in retrospect the location effect was not as shocking. Columbia lower in larger dogs, controlling for the effects of age and sex. Note that weight has been square-root transformed to make visualization easier

samples were collected in EDTA tubes not from the same shipment or brand as the Birmingham and San Antonio samples, yet animals in Columbia had a metabolome more similar to animals in Birmingham. This suggests that sample handling was most likely not the primary reason for the large location effect we witnessed. There are several potential explanations for these findings that immediately come to mind. First, as the dogs were residing in different locations, they were undoubtedly exposed to different gut microbiomes 
Fig. 5 PCA of Columbia dogs with cancer status. Only the positive mode showed some separation of the two groups

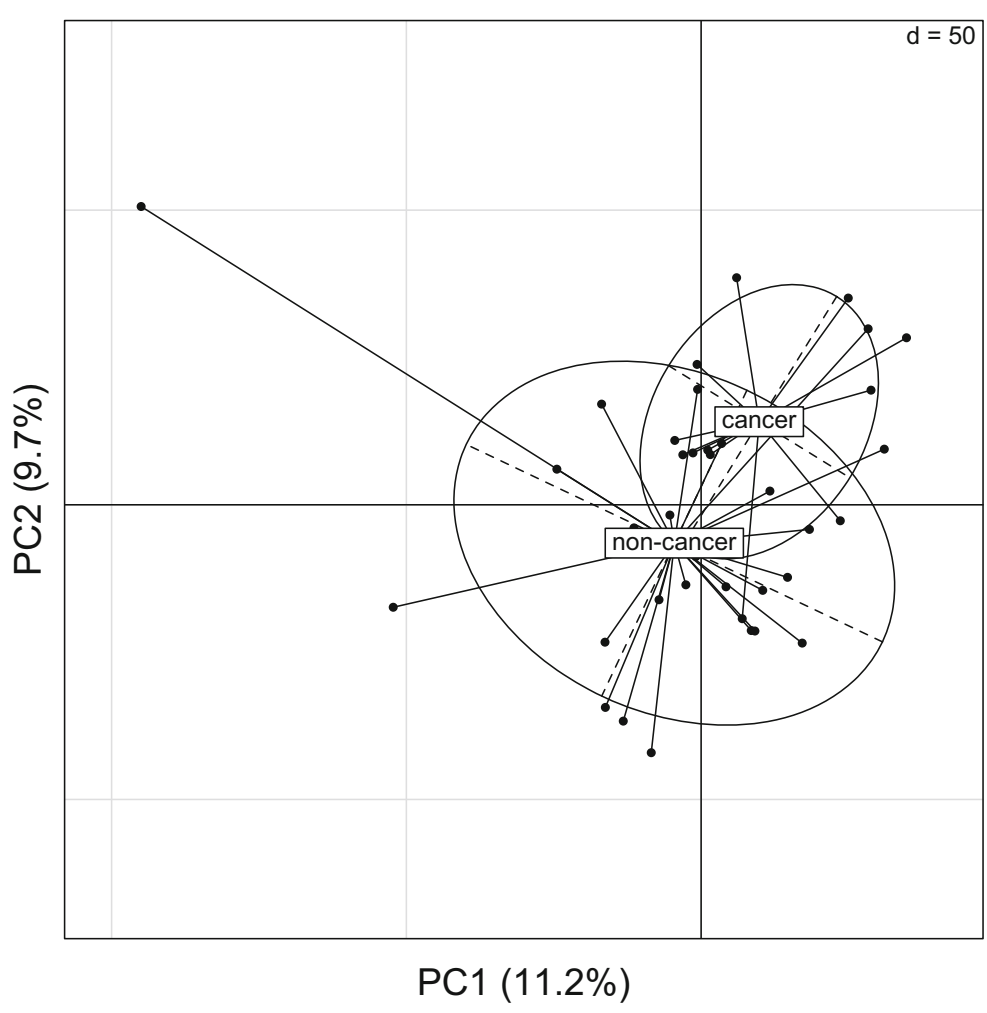

which could be influencing the circulating metabolome in the dogs. Secondly, there could be underlying viruses that do not affect the dog healthwise but do have some physiological effect on the metabolome. This specifically might explain the large differences observed in the San Antonio population. In addition, all dogs from San Antonio were younger, intact, and tended to be smaller which could have biased our metabolomic results. However, sterilization status did not have a significant effect on individual metabolites which is why it was removed from our final linear model analysis. Lastly, there was a difference in time spent fasting for the San Antonio compared with the other two locations as described in the methods. San Antonio dogs were fasted overnight while the same was not true for Birmingham and Columbia dogs. This difference in fasting could have contributed to some of the variations seen in the metabolome across the locations. Recent research in Labrador Retrievers suggests that the fasting plasma metabolome is significantly different from those of recently fed dogs (Soder et al. 2019). However, this study did not look at different periods of fasting. In addition, studies in humans suggest that only certain groups of metabolites (i.e., carnitines) are associated with fasting time (Sedlmeier et al. 2018). Overall, we conclude fasting status could have played a significant but likely minor role in our strong location signals. All these reasons notwithstanding, there are many other possibilities for the observed differences between locations including, but not limited to, different environmental exposures (e.g., diet, air quality, urban/rural residence, climate differences). Most likely, a combination of factors contributes to the strong location effect seen in our metabolomic samples.

Our study suggests that when working with individuals from different locations, especially if these are individuals living in the natural environment not the laboratory, we must control for potential location effects. The advice is most likely applicable to all "omics" in which "levels" of different biological factors are measured (i.e., transcriptomics and proteomics). Accordingly, any future canine studies from which samples are derived from different populations must have location as a controlling effect even if all sample handling procedures are consistent between sites.

Even with all the limitations of using non-laboratory animals in metabolomic profiling, we still find significant metabolites associated with age and weight in both 
our location residual analysis and our Birminghamspecific analysis. Metabolites associated with tryptophan metabolism and fatty acid metabolism were found to be differentially regulated between large and small dogs. This held true when looking at the entire dataset controlling for location effect and in the Birminghamonly dataset (the only dataset for which a large enough number of metabolites passed our FDR to be used for enrichment analyses). Metabolites in the tryptophan metabolism pathway tended to be higher in small dogs compared with large dogs (Figs. 3 and 4). We found no effect of weight on the two major players in the tryptophan metabolism pathway: L-tryptophan and kynurenine. However, no metabolites related to tryptophan metabolism showed a positive associated between weight and metabolite concentration. This pattern supports recent research suggesting that tryptophan metabolism may be an integral part of aging and longevity. High tryptophan (van der Goot and Nollen 2013) and low kynurenine (Sutphin et al. 2017) have been shown to promote longevity in worms, and low tryptophan levels were associated with increased risk of mortality in marmosets (Hoffman et al. 2018b).

The tryptophan metabolic network is complex. Tryptophan is a necessary precursor for the synthesis of the neurotransmitters, serotonin, and melatonin. However, $95 \%$ of dietary tryptophan enters the kynurenine pathway, where an end-product is NAD, a key player in energy metabolism and a co-factor for many enzymes such as sirtuins that are known to be involved in multiple aspects of health and longevity (Mouchiroud et al. 2013). Enhancing physiological NAD via various precursors has been shown to increase longevity and health in multiple species (Zhang et al. 2016). On the other hand, inhibition of the kynurenine pathway — which should reduce NAD - has also been observed to increase health and longevity (van der Goot and Nollen 2013). The resolution of this seeming paradox likely involves bioactive intermediates in the kynurenine pathway such as quinolinic acid which is an NMDA receptor agonist and/or kynurinic acid which is an antagonist of glutamate receptors. The kynurenine pathway also interacts with the mTOR network (Badawy 2017). Dogs, with their range of longevities, may be informative in refining our knowledge of the intricacies of this pathway.

Similar to our results, tryptophan itself was not found to be associated with weight in a previous canine metabolomics study (Middleton et al. 2017); however, they failed to find a signal of tryptophan degradation in their body weight analysis. Changes in tryptophan metabolism have also been shown to be related to diarrhea in dogs (Guard et al. 2015), suggesting changes in tryptophan metabolism might be indicative not just of size but also disease in individual dogs. While these studies combined with ours would suggest that higher tryptophan pathway metabolite levels are beneficial for longevity, previous research has shown that tryptophan restriction increases lifespan in both mice (De Marte and Enesco 1986) and rats (Segall and Timiras 1976). Therefore, the overall contribution of the tryptophan pathway to aging and longevity is still a very much needed area of research. Further interrogation of the tryptophan metabolism pathway is warranted to understand how manipulation may influence size and longevity.

Surprisingly, we failed to find any metabolite associated with weight or age in our Columbia population, even though they were more similar to the Birmingham population when comparing the entire metabolome. This population of dogs came from the Veterinary Health Center at the University of Missouri College of Veterinary Medicine, and as such represented an older, sicker population compared with the other two locations with the majority of dogs sampled in this population having at least one major health concern. These differences in health status are potentially driving the lack of metabolomic associations in the population and would also lend support to our PCA analysis which discovered some separation of a dogs diagnosed with and without cancer (Fig. 5). As older adults often present with at least one major morbidity, the Columbia results suggest that future large metabolomic studies in humans might also fail to find strong age or sex effects due to the overwhelming physiological changes that occur in response to disease as individuals age.

Interestingly, sex was not associated with individual metabolites. None passed our FDR correction threshold across locations, and only San Antonio showed a sex effect of any metabolites in the location-specific models. The San Antonio samples may have been characterized by differences by sex because they were all from intact dogs that were primarily young to middle-aged. Thus, the variation from sterilization and age was much less in this population compared with the other two locations. The overall lack of metabolites associated with sex is consistent with our previous finding that companion dogs do not show sex differences in longevity nor causes of death (Hoffman et al. 2017). However, these 
results are in contrast to the majority of the metabolomic literature which suggests that sex plays a large role on individual metabolites. Potentially, our FDR threshold was too stringent for the small effects of sex, but as we are looking at thousands of metabolites, these differences manifest themselves when combined together.

Our results combined increase knowledge to the overall field of metabolism and aging. Metabolism itself has been strongly linked to aging for more than a century (Austad and Fischer 1991; Rubner 1908). Over the past decade however, attention has focused more on the details of metabolic regulation which has emerged as a major contributor to the aging phenotype. Metabolomic studies have been increasingly used in both model (e.g., Fuchs et al. 2010; Hoffman et al. 2014; Houtkooper et al. 2011) and non-model (Ball et al. 2018; Hoffman et al. 2018b; Hoffman et al. 2016; Lewis et al. 2018; Viltard et al. 2019) organisms, leading to the discovery of common conserved pathways associated with aging and longevity. Also, diet as shown by dietary restriction, the most robust method to increase lifespan and health in model organisms, has been found to profoundly modify metabolic regulation (Matyi et al. 2018). Combined, it is evident that research on metabolic regulation with attention to individual metabolites may provide novel insights into mechanisms underlying the aging phenotype.

While we have presented the largest canine metabolome study to date, our results are not without many limitations due to the nature of sampling companion owned dogs. First, a large proportion of the metabolome is influenced by the environment-specifically the diet - and we do not know what each individual dog ate or was exposed to. However, we would not expect there to be strong differences across the three populations with regard to diet, as it would not be expected that owners in one location would feed a significantly different diet than another location within the USA. We would assume, though our assumption could be incorrect, that the variation in diets is the same across all three populations. While diet most likely does not explain the large differences between populations, diet is most assuredly leading to noise variation in the data. In addition, we lack information on the timing, amount, or type of food consumption for each individual dog, although any or all of these could be contributing to the changes we see in tryptophan metabolism between large and small dogs. Second, circadian rhythms play a large role in metabolomic profiles, and as these were personal owned dogs, we cannot control the time of day that each sample was taken. It was dependent on when the dog was brought into the clinic. Similar to the diet results, time of day of sample collection should not differ between the locations but would add more noise into our collected data. Third, while we do not know why our locations showed significantly different metabolomic profiles, some hypotheses are described above. However, there could have been differences in sample handling between the three locations that we are unaware of that led to the variation seen in the dataset. Lastly, we did not investigate breed-specific differences in our analysis, as we did not have the statistical power to do so with the number of breeds included in the study. Previous research, however, has shown that breeds do have different metabolomic profiles (Liu et al. 2016; Nicholatos et al. 2019), so breed variation in this study is likely to have influenced our metabolomic profiles. However, the wide range of breeds, including mixedbreed dogs, in our analysis actually allows us to better focus on size and age differences, as our results were significant despite the number of breeds represented in our study. Thus, the results reported here are likely not due to breed-specific differences but are more likely due to changes in age and genetic size effects.

\section{Conclusions}

Here, we found a strong effect of location on metabolomic profiles in companion dogs; our results suggest that metabolomic profiles can be strongly influenced by location, and future large "omics" studies need to account for this strong geographic signal. After controlling for location effects, we found a strong signal of tryptophan metabolism and size. Tryptophan pathway metabolites were higher in small, long-lived dogs compared with their large counterparts, and future studies are needed to determine the direct physiological consequences of tryptophan metabolism manipulation. We still have ways to go to fully understand the metabolic differences that are found between large and small dogs, but we have paved the way for future large-scale "omics" studies in the companion dog.

Acknowledgments First and foremost, we would like to thank all the dogs that were sampled during the course of this study and their owners for permitting sampling. We would also like to thank Andrew Brown for insights on the statistical analyses in this manuscript. 
Author Contributions JMH and SNA designed the experiment. JVK, MA, AR, and CH oversaw collection of the blood samples. VT and DPJ ran the metabolomics analysis. JMH analyzed the data and made the figures. JMH and SNA wrote the first draft of the manuscript. All authors commented on and approved the final manuscript.

Funding information This work was funded by a Glenn/AFAR Postdoctoral Fellowship to JMH and NIH/NIA AG050886 and an award from the Glenn Foundation for Medical Research to SNA

Compliance with ethical standards Collection of blood samples from person-owned dogs was approved under UAB IACUC 21121 and MU ACUC 8240.

Open Access This article is distributed under the terms of the Creative Commons Attribution 4.0 International License (http:// creativecommons.org/licenses/by/4.0/), which permits unrestricted use, distribution, and reproduction in any medium, provided you give appropriate credit to the original author(s) and the source, provide a link to the Creative Commons license, and indicate if changes were made.

\section{References}

An JY, Darveau R, Kaeberlein M (2018) Oral health in geroscience: animal models and the aging oral cavity. Geroscience 40:1-10

Austad SN, Fischer KE (1991) Mammalian aging, metabolism, and ecology: evidence from the bats and marsupials. $\mathrm{J}$ Gerontol 46:B47-B53

Badawy AA (2017) Kynurenine pathway of tryptophan metabolism: regulatory and functional aspects. Int J Tryptophan Res 10:1178646917691938

Ball HC, Levari-Shariati S, Cooper LN, Aliani M (2018) Comparative metabolomics of aging in a long-lived bat: insights into the physiology of extreme longevity. PLoS One 13:e0196154

Bartke A (2005) Minireview: role of the growth hormone/insulinlike growth factor system in mammalian aging. Endocrinology 146:3718-3723

Benjamini Y, Hochberg Y (1995) Controlling the false discovery rate - a practical and powerful approach to multiple testing. $\mathrm{J}$ Roy Stat Soc B Met 57:289-300

Brandts L, van den Brandt PA (2019) Body size, non-occupational physical activity and the chance of reaching longevity in men and women: findings from the Netherlands Cohort Study. J Epidemiol Community Health 73:239-249

Cheon DJ, Orsulic S (2011) Mouse models of cancer. Annu Rev Pathol 6:95-119

Darst BF, Koscik RL, Hogan KJ, Johnson SC, Engelman CD (2019) Longitudinal plasma metabolomics of aging and sex. Aging (Albany NY) 11:1262-1282

De Marte ML, Enesco HE (1986) Influence of low tryptophan diet on survival and organ growth in mice. Mech Ageing Dev 36: $161-171$
Dray S, Dufour A (2007) The ade4 package: implementing the duality diagram for ecologists. J Stat Softw 22:1-20

Favier RP, Mol JA, Kooistra HS, Rijnberk A (2001) Large body size in the dog is associated with transient GH excess at a young age. J Endocrinol 170:479-484

Fleming JM, Creevy KE, Promislow DEL (2011) Mortality in North American dogs from 1984 to 2004: an investigation into age-, size-, and breed-related causes of death. J Vet Intern Med 25:187-198

Forster GM, Stockman J, Noyes N, Heuberger AL, Broeckling CD, Bantle CM, Ryan EP (2018) A comparative study of serum biochemistry, metabolome and microbiome parameters of clinically healthy, normal weight, overweight, and obese companion dogs. Top Companion Anim Med 33: 126-135

Fuchs S, Bundy JG, Davies SK, Viney JM, Swire JS, Leroi AM (2010) A metabolic signature of long life in Caenorhabditis elegans. BMC Biol 8

Gookin JL, Mathews KG, Cullen J, Seiler G (2018) Qualitative metabolomics profiling of serum and bile from dogs with gallbladder mucocele formation. PLoS One 13:e0191076

Greer KA, Hughes LM, Masternak MM (2011) Connecting serum IGF-1, body size, and age in the domestic dog. Age (Dordr) 33:475-483

Guard BC, Barr JW, Reddivari L, Klemashevich C, Jayaraman A, Steiner JM, Vanamala J, Suchodolski JS (2015) Characterization of microbial dysbiosis and metabolomic changes in dogs with acute diarrhea. PLoS One 10:e127259

Hasegawa T, Sumita M, Horitani Y, Tamai R, Tanaka K, Komori M, Takenaka S (2014) Gas chromatography-mass spectrometry-based metabolic profiling of cerebrospinal fluid from epileptic dogs. J Vet Med Sci 76:517-522

He Q, Morris BJ, Grove JS, Petrovitch H, Ross W, Masaki KH, Rodriguez B, Chen R, Donlon TA, Willcox DC et al (2014) Shorter men live longer: association of height with longevity and FOXO3 genotype in American men of Japanese ancestry. PLoS One 9:e94385

Healy K, Guillerme T, Finlay S, Kane A, Kelly SB, McClean D, Kelly DJ, Donohue I, Jackson AL, Cooper N (2014) Ecology and mode-of-life explain lifespan variation in birds and mammals. Proc Biol Sci 281:20140298

Hoffman JM, Creevy KE, Franks A, O’Neill DG, Promislow DEL (2018a) The companion dog as a model for human aging and mortality. Aging Cell 17:e12737

Hoffman JM, O’Neill DG, Creevy KE, Austad SN (2017) Do female dogs age differently than male dogs? J Gerontol A Biol Sci Med Sci

Hoffman JM, Ross C, Tran V, Promislow DEL, Tardif S, Jones DP (2018b) The metabolome as a biomarker of mortality risk in the common marmoset. Am J Primatol:e22944

Hoffman JM, Soltow QA, Li SZ, Sidik A, Jones DP, Promislow DEL (2014) Effects of age, sex, and genotype on highsensitivity metabolomic profiles in the fruit fly, Drosophila melanogaster. Aging Cell 13:596-604

Hoffman JM, Tran V, Wachtman LM, Green CL, Jones DP, Promislow DE (2016) A longitudinal analysis of the effects of age on the blood plasma metabolome in the common marmoset, Callithrix jacchus. Exp Gerontol 76:17-24

Houtkooper RH, Argmann C, Houten SM, Canto C, Jeninga EH, Andreux PA, Thomas C, Doenlen R, Schoonjans K, Auwerx 
J (2011) The metabolic footprint of aging in mice. Sci RepUk 1

Jimenez AG, Winward J, Beattie U, Cipolli W (2018) Cellular metabolism and oxidative stress as a possible determinant for longevity in small breed and large breed dogs. PLoS One 13: e0195832

Jin K, Hoffman JM, Creevy KE, O'Neill DG, Promislow DE (2016) Multiple morbidities in companion dogs: a novel model for investigating age-related disease. Pathobiol Aging Age Relat Dis 6:33276

Kaeberlein M, Creevy KE, Promislow DE (2016) The dog aging project: translational geroscience in companion animals. Mamm Genome 27:279-288

Lewis KN, Rubinstein ND, Buffenstein R (2018) A window into extreme longevity; the circulating metabolomic signature of the naked mole-rat, a mammal that shows negligible senescence. Geroscience 40:105-121

Li SZ, Park Y, Duraisingham S, Strobel FH, Khan N, Soltow QA, Jones DP, Pulendran B (2013) Predicting network activity from high throughput metabolomics. PLoS Comput Biol 9: e1003123

Liu KH, Walker DI, Uppal K, Tran V, Rohrbeck P, Mallon TM, Jones DP (2016) High-resolution metabolomics assessment of military personnel: evaluating analytical strategies for chemical detection. J Occup Environ Med 58:S53-S61

Lloyd AJ, Beckmann M, Tailliart K, Brown WY, Draper J, Allaway D (2016) Characterisation of the main drivers of intra- and inter- breed variability in the plasma metabolome of dogs. Metabolomics 12:72

Ma W, Hagan KA, Heianza Y, Sun Q, Rimm EB, Qi L (2017) Adult height, dietary patterns, and healthy aging. Am J Clin Nutr 106:589-596

Matyi S, Jackson J, Garrett K, Deepa SS, Unnikrishnan A (2018) The effect of different levels of dietary restriction on glucose homeostasis and metabolic memory. Geroscience 40:139149

Menni C, Kastenmuller G, Petersen AK, Bell JT, Psatha M, Tsai PC, Gieger C, Schulz H, Erte I, John S et al (2013) Metabolomic markers reveal novel pathways of ageing and early development in human populations. Int J Epidemiol 42: 1111-1119

Middleton RP, Lacroix S, Scott-Boyer MP, Dordevic N, Kennedy AD, Slusky AR, Carayol J, Petzinger-Germain C, Beloshapka A, Kaput J (2017) Metabolic differences between dogs of different body sizes. J Nutr Metab 2017: 4535710

Miller RA, Harper JM, Galecki A, Burke DT (2002) Big mice die young: early life body weight predicts longevity in genetically heterogeneous mice. Aging Cell 1:22-29

Minamoto Y, Otoni CC, Steelman SM, Buyukleblebici O, Steiner JM, Jergens AE, Suchodolski JS (2015) Alteration of the fecal microbiota and serum metabolite profiles in dogs with idiopathic inflammatory bowel disease. Gut Microbes 6:3347

Mouchiroud L, Houtkooper RH, Moullan N, Katsyuba E, Ryu D, Canto C, Mottis A, Jo YS, Viswanathan M, Schoonjans K et al (2013) The NAD(+)/sirtuin pathway modulates longevity through activation of mitochondrial UPR and FOXO signaling. Cell 154:430-441

Nicholatos JW, Robinette TM, Tata SVP, Yordy JD, Francisco AB, Platov M, Yeh TK, Ilkayeva OR, Huynh FK, Dokukin M,
Volkov D, Weinstein MA, Boyko AR, Miller RA, Sokolov I, Hirschey MD, Libert S (2019) Cellular energetics and mitochondrial uncoupling in canine aging. Geroscience 41:229 242

O'Neill DG, Church DB, McGreevy PD, Thomson PC, Brodbelt DC (2013) Longevity and mortality of owned dogs in England. Vet J 198:638-643

Rollo CD (2002) Growth negatively impacts the life span of mammals. Evol Dev 4:55-61

Rubner M (1908) Das Problem det Lebensdaur und seiner beziehunger zum Wachstum und Ernarnhung. Oldenburg, Munich

Sedlmeier A, Kluttig A, Giegling I, Prehn C, Adamski J, Kastenmuller G, Lacruz ME (2018) The human metabolic profile reflects macro- and micronutrient intake distinctly according to fasting time. Sci Rep 8:12262

Segall PE, Timiras PS (1976) Patho-physiologic findings after chronic tryptophan deficiency in rats: a model for delayed growth and aging. Mech Ageing Dev 5:109-124

Soder J, Hagman R, Dicksved J, Lindase S, Malmlof K, Agback P, Moazzami A, Hoglund K, Wernersson S (2017) The urine metabolome differs between lean and overweight Labrador Retriever dogs during a feed-challenge. PLoS One 12: $\mathrm{e} 0180086$

Soder J, Hoglund K, Dicksved J, Hagman R, Eriksson Rohnisch H, Moazzami AA, Wernersson S (2019) Plasma metabolomics reveals lower carnitine concentrations in overweight Labrador Retriever dogs. Acta Vet Scand 61:10

Soltow QA, Strobel FH, Mansfield KG, Wachtman L, Park Y, Jones DP (2013) High-performance metabolic profiling with dual chromatography-Fourier-transform mass spectrometry (DC-FTMS) for study of the exposome. Metabolomics 9: S132-S143

Sutphin GL, Backer G, Sheehan S, Bean S, Corban C, Liu T, Peters MJ, van Meurs JBJ, Murabito JM, Johnson AD et al (2017) Caenorhabditis elegans orthologs of human genes differentially expressed with age are enriched for determinants of longevity. Aging Cell 16:672-682

Tapprest J, Morignat E, Dornier X, Borey M, Hendrikx P, Ferry B, Calavas D, Sala C (2017) Fallen stock data: an essential source of information for quantitative knowledge of equine mortality in France. Equine Vet J 49:596-602

Team RCD (2018) R: A language and environment for statistical computing. Version 3.5.2. In R Foundation for Statistical Computing. Vienna, Austria

Uppal K, Soltow QA, Strobel FH, Pittard WS, Gernert KM, Yu TW, and Jones DP (2013) xMSanalyzer: automated pipeline for improved feature detection and downstream analysis of large-scale, non-targeted metabolomics data. Bmc Bioinformatics 14.

Urfer SR, Kaeberlein TL, Mailheau S, Bergman PJ, Creevy KE, Promislow DE, Kaeberlein M (2017a) Asymptomatic heart valve dysfunction in healthy middle-aged companion dogs and its implications for cardiac aging. Geroscience 39:43-50

Urfer SR, Kaeberlein TL, Mailheau S, Bergman PJ, Creevy KE, Promislow DEL, Kaeberlein M (2017b) A randomized controlled trial to establish effects of short-term rapamycin treatment in 24 middle-aged companion dogs. Geroscience 39: $117-127$ 
van der Goot AT, Nollen EA (2013) Tryptophan metabolism: entering the field of aging and age-related pathologies. Trends Mol Med 19:336-344

Viant MR, Ludwig C, Rhodes S, Gunther UL, Allaway D (2007) Validation of a urine metabolome fingerprint in dog for phenotypic classification. Metabolomics 3:453-463

Viltard M, Durand S, Perez-Lanzon M, Aprahamian F, Lefevre D, Leroy C, Madeo F, Kroemer G, Friedlander G (2019) The metabolomic signature of extreme longevity: naked mole rats versus mice. Aging (Albany NY) 11:4783-4800

von Scheidt M, Zhao Y, Kurt Z, Pan C, Zeng L, Yang X, Schunkert H, Lusis AJ (2017) Applications and limitations of mouse models for understanding human atherosclerosis. Cell Metab 25:248-261
Yu TW, Park Y, Johnson JM, Jones DP (2009) apLCMS-adaptive processing of high-resolution LC/MS data. Bioinformatics 25:1930-1936

Zhang H, Ryu D, Wu Y, Gariani K, Wang X, Luan P, D'Amico D, Ropelle ER, Lutolf MP, Aebersold R, Schoonjans K, Menzies KJ, Auwerx J (2016) NAD(+) repletion improves mitochondrial and stem cell function and enhances life span in mice. Science 352:1436-1443

Publisher's note Springer Nature remains neutral with regard to jurisdictional claims in published maps and institutional affiliations. 